\title{
Renal Sparing Surgery for Perirenal Liposarcoma: 24 Months Recurrence Free Follow Up
}

\author{
Narmada P. Gupta, Rajiv Yadav \\ Department of Urology, All India Institute of Medical Sciences, New Delhi, India
}

\begin{abstract}
Liposarcoma is the most common pathology seen in the soft tissue sarcoma of retroperitoneum. These tumors have been traditionally treated with radical surgery sacrificing adjacent organs to achieve clear margins. We have reported our experience of renal sparing surgery for perirenal liposarcoma in two patients with more than 24 month disease free survival.
\end{abstract}

Key words: retroperitoneal neoplasm; liposarcoma; surgery

Int Braz J Urol. 2007; 33: 181-92

\section{INTRODUCTION}

Soft-tissue sarcomas of the retroperitoneum are relatively uncommon tumors with an incidence of 2 in 100,000 (1). Liposarcoma is the most common pathology seen in the soft tissue sarcoma of retroperitoneum (61\%); $79 \%$ of which are low grade (2). In general, the biological behavior and prognosis of liposarcomas (LPS) are more favorable compared to most soft tissue sarcomas (STS) but the prognosis can vary widely depending on tumor characteristics, especially histological subtype and tumor grade (3). Emphasizing the importance of radical resection and tumor negative surgical margins for disease specific survival, these tumors have traditionally been treated with radical surgery sacrificing adjacent organs $(1,3-$ 5). Multiple trials of chemotherapy and radiation therapy have shown no survival benefit. We report our experience of renal sparing surgery for perirenal liposarcoma in two patients with an excellent postoperative outcome and more than 24 month disease free survival.

\section{CASES PRESENTATION}

Case 1: A fifty-year diabetic and hypertensive male presented with a lump in the left side of upper abdomen and 3-month dull aching pain of. He had no history of any lower urinary tract symptoms, lithiasis or operative intervention in past. His blood sugar and blood pressure was normal with oral medication. He had normal renal function and hemogram. Computed tomography of abdomen and pelvis revealed a $12 \times 10$ centimeter mass with lipomatous density involving the perirenal fat without infiltrating surrounding organs.

Case 2: Fifty seven year old woman presented with a 2-month right flank pain with no other urinary symptoms. Clinically examination revealed right hypochondrial renal swelling. Computed tomography of abdomen and pelvis revealed $10 \times 8$ centimeter perinephric lesion with fat attenuation surrounding the upper and mid polar region.

The preoperative imaging study was suggestive of a close association of tumor with the 
kidneys in both the cases (Figures-1 and 2) but intraoperative assessment resulted a plan of renal sparing approach in both the situations. Intraoperatively, a well encapsulated lipomatous tumor was found to extend from the upper pole to the lower pole draping the surface of the renal capsule and infiltrating the renal capsule in one of the locations over $1 \mathrm{~cm}$ diameter region (Figures-3 and 4). Complete tumor resection was achieved after wedge resection of renal parenchyma with $1 \mathrm{~cm}$ margin.
Histopathology revealed well-differentiated liposarcoma with focal invasion of the renal capsule in both cases (Figure-5). Dissected margins were free of tumor.

Both patients were discharged after 72 hours postoperatively without any complication. Patients were kept on a three monthly follow-up. Computed tomography images were repeated at 6 month intervals for two years. At present, both patients are completely asymptomatic, free of local recurrence (Figure-6) or distant metastases.

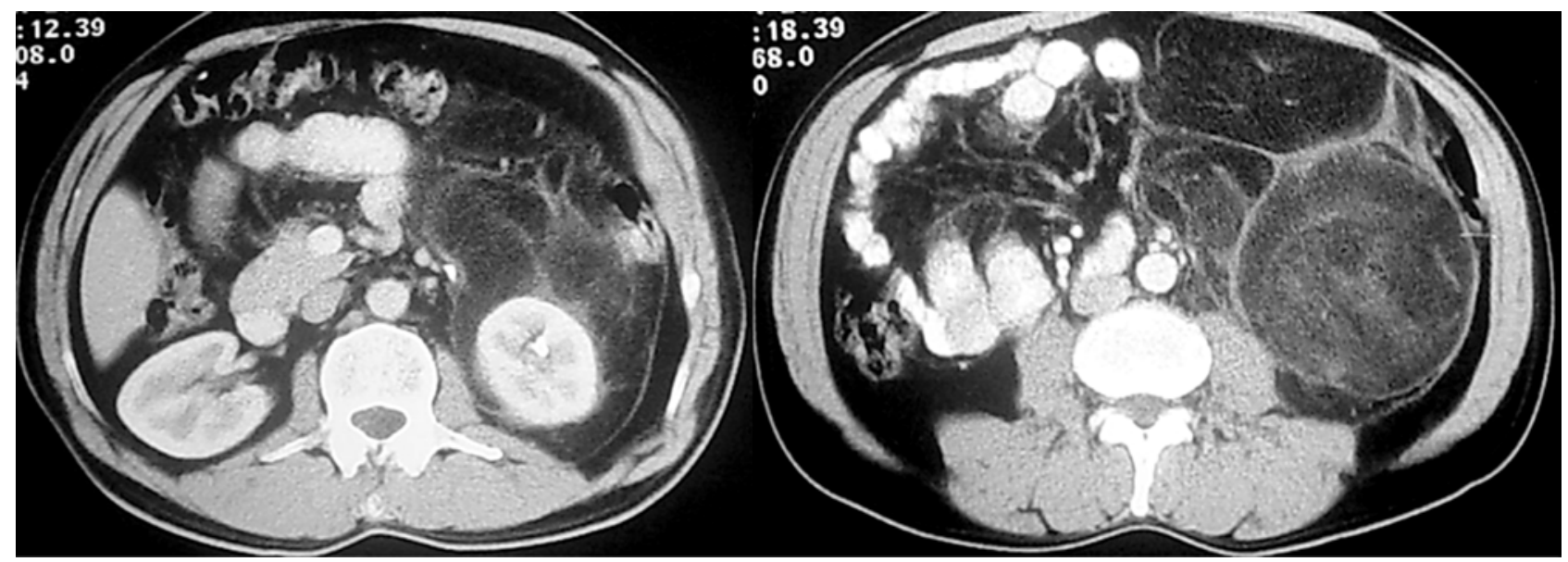

Figure 1 - Large perinephric tumor with fat attenuation surrounding the left kidney (Case-1).

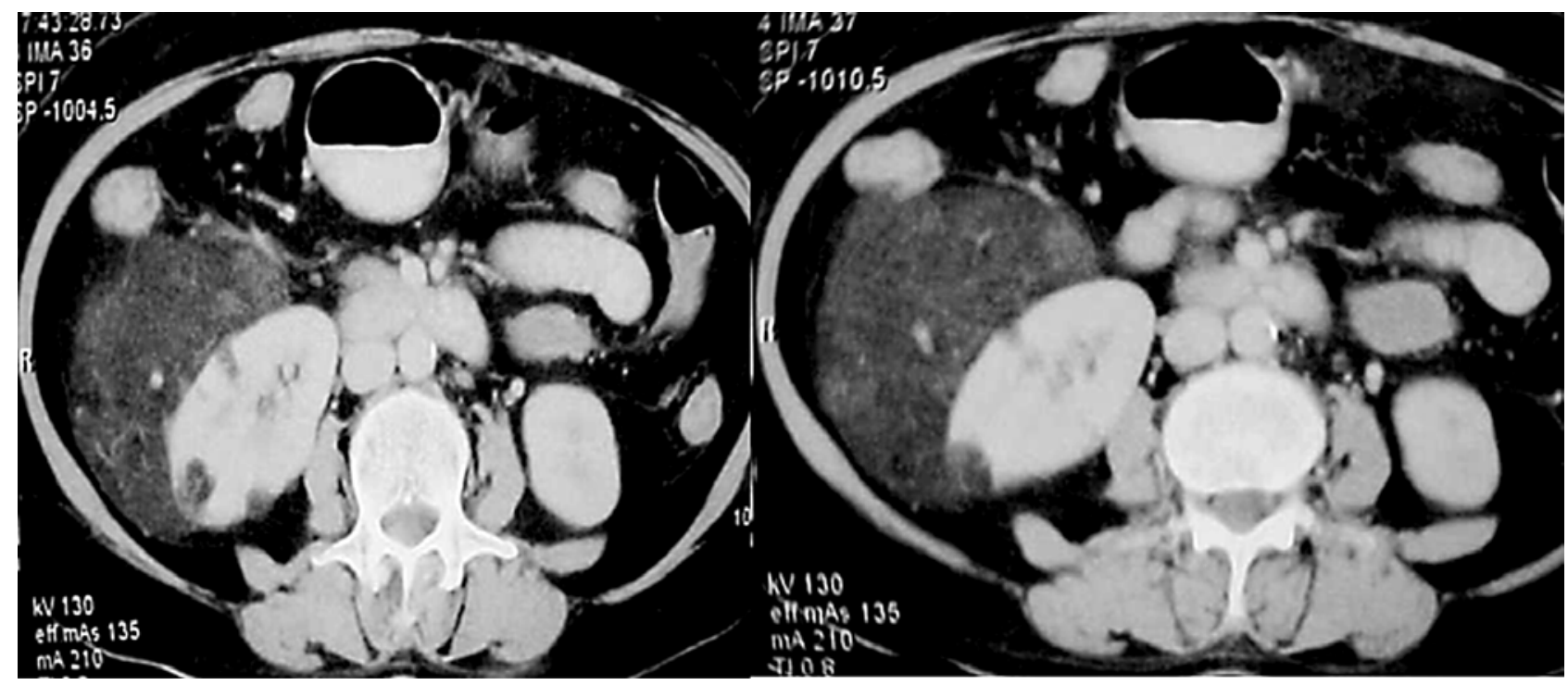

Figure 2 - Perinephric tumor with fat attenuation in relation with the right kidney (Case-2). 

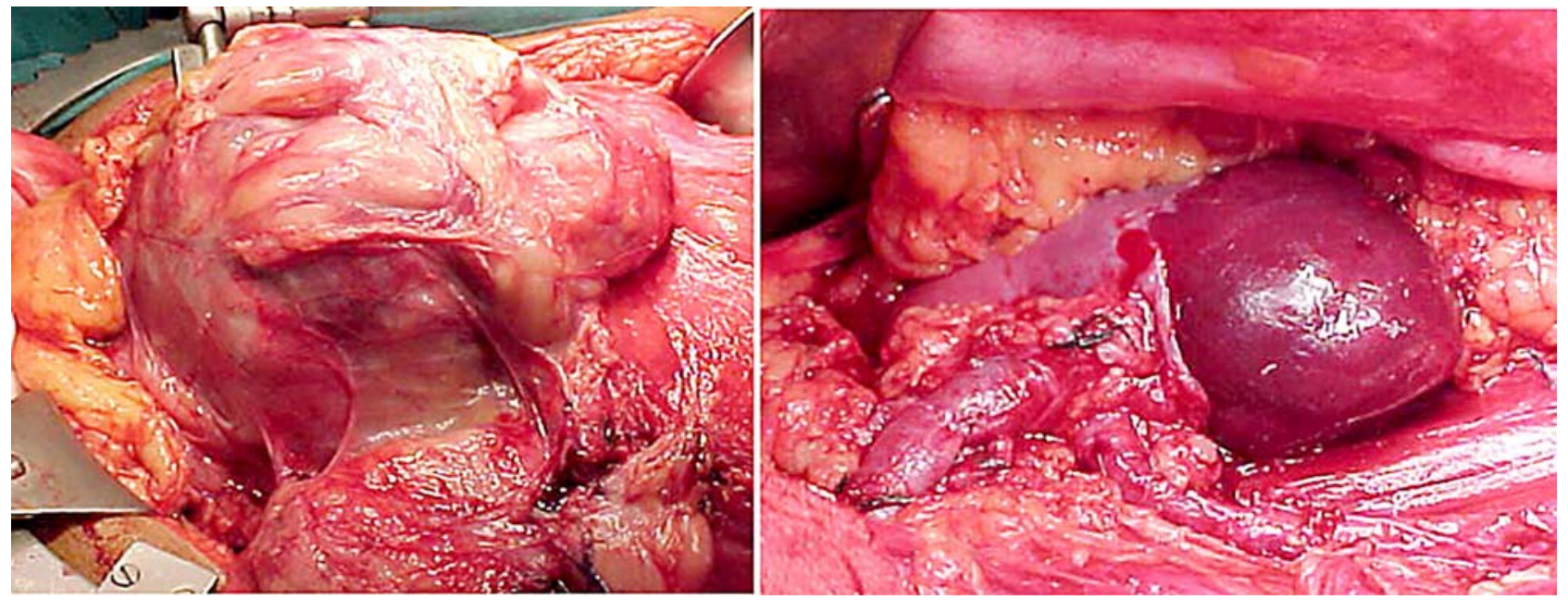

Figure 3 - Intraoperative view of case 1 (before and after removal) showing encapsulated tumor overlying and surrounding the kidney.
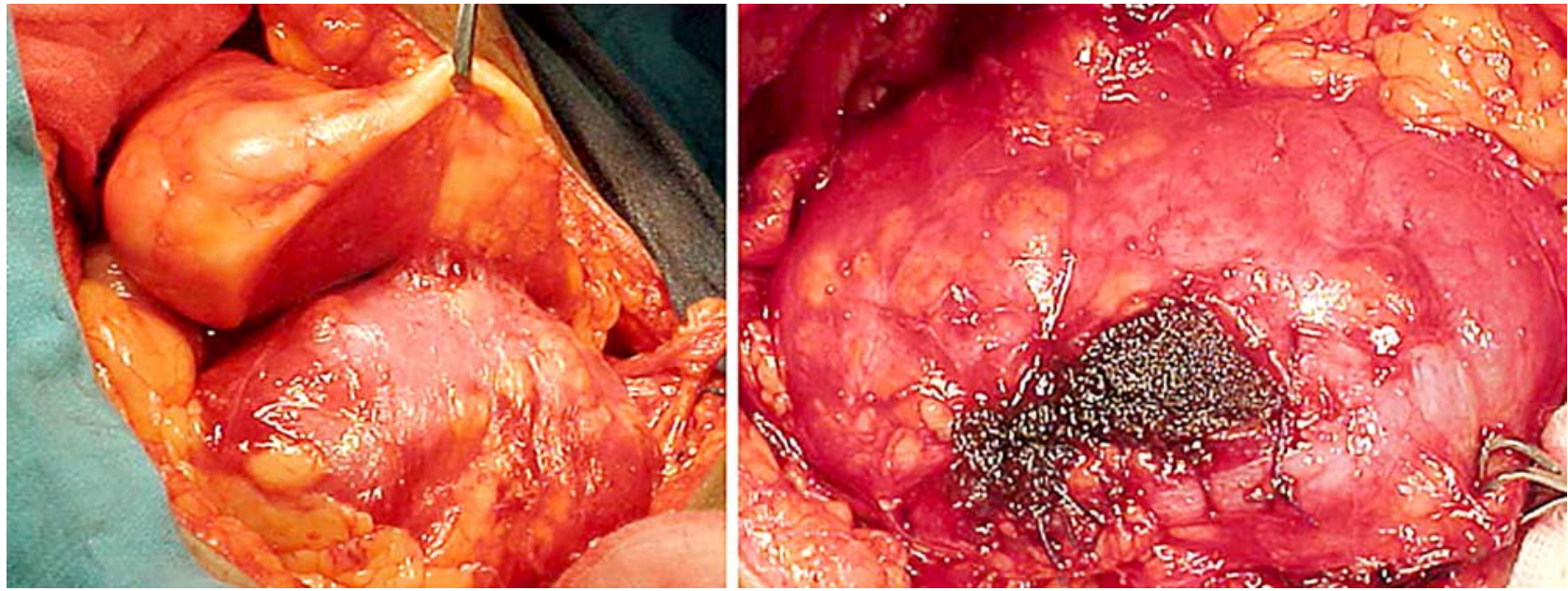

Figure 4 - Intraoperative view of case 2 (before and after removal) showing encapsulated tumor fixed to the kidney.

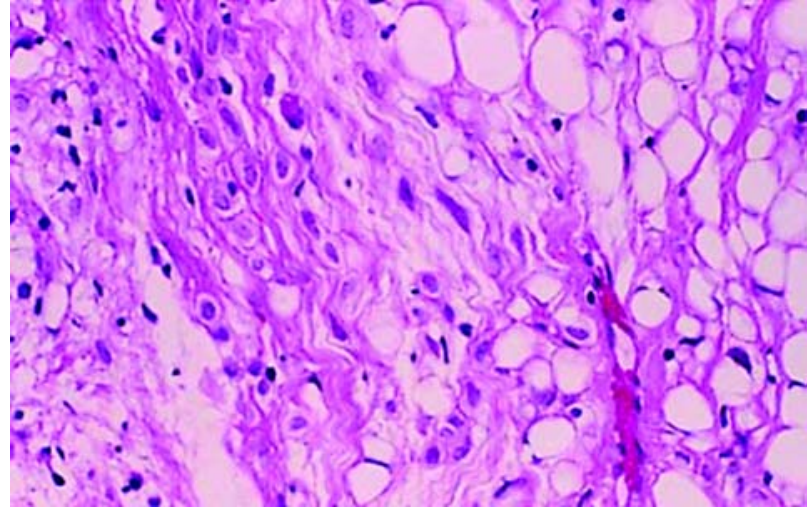

Figure 5 - Histology of the tumor showing well differentiated liposarcoma with areas of atypical cells (arrow).

\section{COMMENTS}

Subsequent to the first description of renal sparing surgery for treatment of renal tumor in 1890 , considerable advances in renal imaging and surgical experience has resulted in good long term survival with such treatment in appropriately selected patients. Regarding the management of retroperitoneal liposarcoma, surgery plays a dominant role in the initial and subsequent treatment. Gronchi et al. (6), in a retrospective study involving 167 consecutive patients who underwent surgery for retroperitoneal soft tissue sarcoma over a 20 year span, assessed long-term 


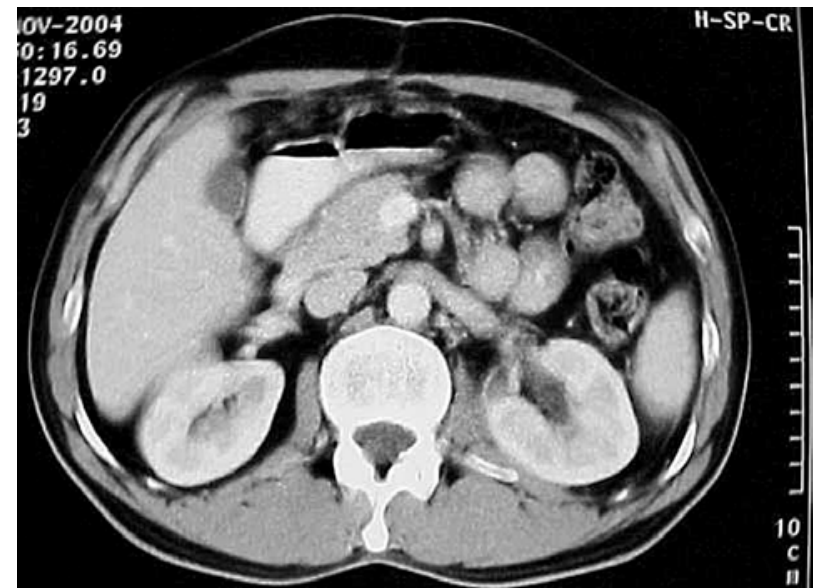

Figure 6 - Follow up computed tomography image of case 1 at two years without any local recurrence.

prognosis and patterns of failure in these patients. Only a minority of patients developed distant metastases. Histological subtype appeared to influence the pattern of recurrence, which was mainly local for patients with liposarcoma. Several authors have noted that tumor negative surgical margins are associated with a reduced risk of local recurrence and improved disease specific survival, especially in patients with low-grade retroperitoneal LPS $(1,3,4)$. In both our case only limited involvement of renal parenchyma was observed at the time of surgery, which made renal sparing surgery a feasible option without compromising the oncological principle of tumor free margins. We believe that the principle of achieving the tumor free margins may be respected by renal sparing surgery in select cases of liposarcoma. The decision to perform nephrectomy should be made based on intraoperative findings rather than solely on preoperative imaging studies.

In conclusion, renal sparing approach in perirenal liposarcoma is a feasible option in select cases with good recurrence free survival.

\section{CONFLICT OF INTEREST}

None declared.

\section{REFERENCES}

1. Voros D, Theodorou D, Ventouri K, Prachalias A, Danias N, Gouliamos A: Retroperitoneal tumors: do the satellite tumors mean something? J Surg Oncol. 1998; 68: 30-3.

2. Bautista N, Su W, O'Connell TX: Retroperitoneal softtissue sarcomas: prognosis and treatment of primary and recurrent disease. Am Surg. 2000; 66: 832-6.

3. Nijhuis PH, Sars PR, Plaat BE, Molenaar WM, Sluiter WJ, Hoekstra HJ: Clinico-pathological data and prognostic factors in completely resected AJCC stage I-III liposarcomas. Ann Surg Oncol. 2000; 7: 535-43.

4. Malerba M, Doglietto GB, Pacelli F, Carriero C, Caprino P, Piccioni E, et al: Primary retroperitoneal soft tissue sarcomas: results of aggressive surgical treatment. World J Surg. 1999; 23: 670-5.

5. Neuhaus SJ, Barry P, Clark MA, Hayes AJ, Fisher C, Thomas JM: Surgical management of primary and recurrent retroperitoneal liposarcoma. Br J Surg. 2005; 92: 246-52.

6. Gronchi A, Casali PG, Fiore M, Mariani L, Lo Vullo S, Bertulli R, et al.: Retroperitoneal soft tissue sarcomas: patterns of recurrence in 167 patients treated at a single institution. Cancer. 2004; 100: 2448-55.

\section{Correspondence address:}

Dr. Narmada P Gupta

Head, Department of Urology

All India Institute of Medical Sciences

Ansari Nagar, New Delhi, 110029, India

E-mail: narmadagupta@ hotmail.com 


\section{EDITORIAL COMMENT}

This is a case report of renal sparing surgery for liposarcoma. The conclusion that this surgery is feasible in selected cases may be premature based on two cases with two year follow-up.

\section{EDITORIAL COMMENT}

The concept is interesting only because it relates to a tumor rarely encountered. The goal with surgery for retroperitoneal sarcomas is complete resection at the time of surgery. Sparing of organs is important as long as oncological principles are not compromised.

Dr. R. Houston Thompson

Professor of Urology

Mayo Medical School, Mayo Clinic

Rochester, Minnesota, USA

E-mail: thompson.robert@mayo.edu

\section{EDITORIAL COMMENT}

This is an interesting report of two patients who underwent nephron-sparing surgery to effect excision of a perirenal liposarcoma. The unusual application of nephron-sparing in this setting renders this report novel and of interest to urological practitioners. The short-term outcome in these patients appears to have been satisfactory with no evidence of tumor recurrence at two years postoperatively.

Dr. Andrew C. Novick

Chair of Urology, Glickman Urological Institute Cleveland Clinic Foundation

Cleveland, Ohio, USA

E-mail:novicka@ccf.org 